\title{
Et si Janet était plus actuel que Freud ?
}

\author{
What if Janet were more up-to-date than Freud?
}

\author{
L.-S. Oulahbib \\ (C) Springer-Verlag 2009
}

Résumé Revenir à l'œuvre de Pierre Janet veut précisément combler une demande, celle qui ne semble pas trouver dans la psychanalyse freudienne les réponses à ces questions qui s'interrogent sur ce qui déclenche un sentiment, sa signification pour la conscience qui s'en saisit, ce qui s'en déduit comme conduite, en quoi la croyance en sa légitimité renforce celle-ci jusqu'à devenir cette force d'impulsion (à ne pas confondre avec la pulsion) qui permet l'effort d'action. Son dérèglement, la pulsion précisément, montre, elle, comment dérivations puis dissociations peuvent un jour se chevaucher lorsque l'impuissance à agir boucle sans fin et vient se réfugier dans des conduites refuges, ou la névrose, selon Janet. Ce dernier déploie ainsi toute une analyse qu'il serait hautement judicieux d'en découvrir à nouveau l'actualité heuristique majeure. Et, à l'heure actuelle, précisément, il existe bel et bien une redécouverte de ce savant, déjà bien entamée dans le monde entier, en particulier au Japon, en Allemagne, au Canada, en Hollande, en Russie, aux États-Unis : il manque la France...

Mots clés Sentiment - Effort · Confiance - Impulsion · Dérivation · Dissociation · Névrose

\begin{abstract}
A return to the work of Pierre Janet is prompted by the desire to satisfy a need as yet unfulfilled by Freudian analysis, addressing such questions as: what triggers a feeling, what does that feeling mean to the consciousness, what behavior does it trigger and how does belief in the feeling's legitimacy strengthen the momentum of the behavior until it enables an active effort (not to be confused with an impulse), while its disturbance, (which is in fact an impulse), shows how derivations and dissociations can overlap, resulting in immobility and escape behavior, or neurosis according to Janet. It would be extremely wise to rediscover the heuristic relevance of Janet's analysis today.
\end{abstract}

\footnotetext{
L.-S. Oulahbib $(\bowtie)$

Docteur en sociologie, habilité à diriger des recherches en sciences politiques, chargé de cours à Lyon 3 et Paris X

86, quai Pierre Scize, 69005, Lyon, France

e-mail : lucien.oulahbib@free.fr
}

And this is exactly what is happening at the moment all over the world, particularly in Japan, Germany, Canada, Holland, Russia and the USA: not in France, however...

Keywords Feeling · Effort · Trust · Impulse ·

Derivation $\cdot$ Dissociation $\cdot$ Neurosis

\section{Délimitation de la méthode janétienne}

\section{Le normal et le pathologique}

Pierre Janet a toujours considéré que la force psychologique ne pouvait être réduite à son aspect neurophysiologique. Dans ces conditions, la thérapie janétienne consiste à renforcer la conscience dans sa capacité à synthétiser, à hiérarchiser l'action, en un mot, à croire en elle de façon réfléchie, tout en la soulageant dans cet effort, en la délestant des décisions lourdes, afin que peu à peu, la conscience puisse déjà reprendre confiance en ses capacités, en ses buts, bref, en la croyance d'être celui-ci et non un autre. Pour Pierre Janet, le normal exprimerait ce moment où la tension ou force se concentre en hiérarchisant les intentions ou tendances à agir, tout en voyant surgir par moments le conflit, la rêverie, lorsque la tension a été trop longtemps maintenue dans une seule direction. Tandis que le pathologique exprime ce moment où la conscience ne peut plus hiérarchiser, où les tendances semblent devenir indépendantes, à la façon de ces gens qui se parlent à eux-mêmes ou semblent plonger dans une torpeur, insensible à tout. En un mot, le pathologique pousse à l'excès ce qui existe à l'état normal, ce qui implique une différence de degré et non de nature entre normal et pathologique et donc requiert une analyse d'ensemble de la Psyché. Ce qui se différencie avec le réductionnisme mécaniste freudien.

\section{Première différence entre Freud et Janet : la méthode}

Freud est mécaniste en ce qu'il s'appuie sur le comparatif appareil psychique, appareil thermique qui, en perte de chaleur, est prêt à en chercher n'importe où pour compenser [1] : d'où l'explication de l'inceste, dont le refoulement crée 
le trauma ; or, si l'on prend plutôt comme base générique moyenne des traumas, l'impuissance à agir, la peur de l'action, comme le fait Janet, la notion de refoulement, que Janet trouvait pertinente, est sauvée et peut alors expliquer de façon multiforme et non pas univoque les accumulations de stress, de tics, d'actes manqués, de phobies, d'obsession, etc., qui, à force, basculent dans la dissociation et forment ainsi l'inconscient, c'est-à-dire dépassent le subconscient pour aller vers le dédoublement de personnalité...

\section{Revanche posthume de Janet sur Freud ?}

Le sociologue, Alain Ehrenberg, dans La Fatigue d'être soi [2], énonce qu'entre Freud et Janet, l'héritage de ce dernier « est peut-être bien plus déterminant pour le sujet qui nous occupe » [3], à savoir les liens entre dépression et société ; une interaction qui lui semble, en effet, aujourd'hui cruciale pour comprendre le psychisme contemporain. Il parle même de « revanche posthume de Janet sur Freud » [4].

C'est que Janet avait principalement mis l'accent sur la relation entre action-effort-personnalité, parce que ces trois éléments se tiennent et s'entretiennent [5]. Cette relation se double néanmoins d'une crainte de l'action elle-même [6] surtout lorsqu'elle s'avère trop transformatrice (comme le faisait remarquer à Janet l'écrivain Raymond Roussel [7], alias Martial [8]). D'où parfois l'angoisse [9], la fatigue prématurée ou, à l'opposé, la frénésie de l'agitation à l'annonce pourtant d'une bonne nouvelle, celle d'une action qui nous a été confiée [10], le tout débouchant sur ces oscillations où alternent euphorie et dépression.

La crainte d'atteindre la plénitude d'action, pourtant désirée, peut avoir diverses raisons; leurs causes n'ont cependant pas à être seulement réduites systématiquement à certaines données affectives ; parce qu'il se peut qu'il ne s'agisse pas, nécessairement, que de traumatisme, a fortiori infantile, ou d'interdits mais aussi d'éducation, d'apprentissage, de mal formation, et ce, surtout peut-être, par exemple, lorsque cette crainte ne s'avère pas systématique ou s'avère compréhensible du fait d'une action exceptionnelle à réaliser, comme prendre la parole devant une foule, diriger une grande entreprise, une nation. La difficulté d'y arriver ne peut à l'évidence se cantonner au seul fait du traumatisme infantile...

\section{Janet comme fondateur de l'analyse multicausale en psychologie}

Contrairement à ce que prétend Elisabeth Roudinesco, Pierre Janet est le réel fondateur de l'analyse psychologique, qu'il nommait également psychologie des conduites [11], et qui consiste à étudier tout ce qui entrave l'action en observant ce que notre corps affiche comme expressions et comportements, en fonction des situations, mais aussi, lorsqu'il en occulte certains aspects. Car, comme il a été dit plus haut, à la différence de la psychologie freudienne, l'analyse psychologique janétienne (fondée par Pierre Janet en 1885), ne base pas tous les troubles psychiques sur le traumatisme sexuel ou le (seul) refoulé [12] ; non pas parce que cela n'existerait pas ou que l'idée même en serait inconvenante selon certains critères moraux (comme le pérore, avec une certaine malhonnêteté intellectuelle, Roudinesco à l'encontre de Janet), ou encore que la suprématie d'un facteur sur tous les autres serait par trop unilatérale, mais bien plutôt parce que c'est, tout d'abord, l'idée même du traumatisme qu'il s'agit de considérer (en ce sens, la méthode janétienne continue celle de Charcot alors que Freud s'en détache).

Il s'agit, dans ce cas, d'orienter l'analyse causale plutôt vers la baisse de la tension psychologique parce qu'elle semble plus fructueuse pour expliquer le symptôme sans doute le plus crucial : la difficulté à agir, accompagnée de ces diverses dérivations qui permettent ou, du moins, signalent la nécessité de la compenser. La psychologie janétienne diagnostiquera alors cela : l'obsession systématique ou névrose qui peut en résulter désigne une difficulté à agir en pleine confiance, d'où la régression vers des formes antérieures d'appréhension de soi.

La thérapie consistera avions-nous dit plus haut à faire croire à nouveau en soi en reconstituant le sentiment de l'effort et la capacité à en hiérarchiser les priorités. Ce qui implique de distinguer le subconscient de l'inconscient : le premier terme désigne l'existence de motivations ou tendances multiples dont l'ensemble forme le Moi (ou Synthèse) et le fait que chacune veut avoir le privilège de conduire l'action, ce qui ne va pas sans conflit qui se manifeste par des incohérences, des actions en sourdine (parfois bénéfiques comme certaines rêveries), ou alors non voulues consciemment comme les maladresses et les distractions.

L'inconscient chez Janet désigne le fait que le conflit entre les motivations ne se trouve pas surmonté. Une synthèse s'avère impossible entre le jeu du subconscient où coexistent toutes les tendances, et le conscient qui doit les hiérarchiser :

Ce qui caractérise la subconscience, ce n'est pas que la tendance diminue ou reste latente, c'est au contraire que les tendances se développent, se réalisent fortement sans que les autres tendances de l'esprit soient averties de leur réalisation et sans qu'elles puissent travailler à s'y opposer [13].

Par ailleurs :

Il y aurait, je crois, toute une étude psychologique des plus curieuses à faire sur cette rêverie intérieure et continuelle qui joue chez beaucoup d'hommes un rôle considérable. On pourrait étudier le contenu de ces rêveries; on y verrait quelquefois de curieux travaux 
psychologiques qui s'effectuent en nous à notre insu. C'est grâce à ce travail subconscient que nous trouvons tout résolus des problèmes que peu de temps auparavant nous ne comprenions pas. C'est ainsi, bien souvent, que nous préparons un livre ou une leçon qui, un beau jour, nous apparaissent tout faits sans que nous comprenions ce miracle. On pourrait aussi, suivant la nature de cette rêverie humble ou ambitieuse, triste ou satisfaite, découvrir bien des lois du caractère et bien des prédispositions cachées [14].

Le conflit entre les tendances s'aggrave de telle façon que des symptômes apparaissent et affichent des tics et des tremblements sans que le sujet n'en ait aucune conscience, jusqu'à rendre insensible une partie de lui-même (microparalysies), qu'il s'agisse d'un organe ou d'un sentiment ou comme ces gens qui se mettent à parler fort en permanence comme s'ils étaient hantés par plusieurs personnalités, ce que Janet nomme la dissociation.

\section{Différence avec l'acception freudienne}

L'acception freudienne de l'inconscient condense, elle, tout ce processus et sa différenciation dissociative en une seule topique (le ça), tout en le basant toujours uniquement sur un seul traumatisme, le sexuel, ce qui a sans doute plu à l'époque où parler de sexe était encore tabou mais apparaît aujourd'hui réducteur, tant le trouble ou l'insatiable désir de s'affirmer, y compris sexuellement, peut, en tant qu'indication et compensation de tension, provenir d'autres facteurs comme, par exemple, un manque de reconnaissance sociale, d'amour (familial, affectif) qui approuve et encourage...

En un mot, si l'enfant mâle sent la confiance de sa mère, il se croira en mesure non pas de lui faire l'amour mais de plaire aux autres femmes qu'elle. De même, lorsque dans la rue des personnes se jaugent de ce regard miroir qui symbolise le désir d'être reconnu, il s'agit moins d'y voir une séduction débridée qu'un effort cherchant à se conforter dans la perdurance de son être. C'est donc bien la croyance en soi qui est le facteur premier des névroses, c'est-à-dire, pour Janet, le fait de ne pas accepter d'évoluer, et donc, d'en rester uniquement à certaines habitudes acquises souvent dans l'enfance.

\section{Effort comme conduite}

L'action, qu'elle soit primaire ou secondaire [15] (c'est-àdire élémentaire ou réflexive), et ce, en interne ou en externe [16], nécessite d'autant plus un effort, qu'elle s'avère nouvelle ; l'effort étant conçu bien plus comme une concentration d'attentions qu'une force proprement organique [17], autrement dit, il s'agit d'une conduite [18], à savoir l'organisation synthétique de décisions en vue de l'action, qu'il s'agit d'accompagner par un « mental » bien organisé. Cette apparente trivialité déploie ses écueils chaque jour. Surtout lorsque l'effort est inédit, parce qu'il s'agit d'intégrer de nouvelles données et de forger des automatismes correspondants ; ce qui est coûteux en termes d'énergie (et cela ne veut pas dire nécessairement de la force, répétons-le, mais de l'attention par exemple) ; et cela peut être déroutant, puisque l'on ne va pas émettre des sentiments de la même façon. C'est-à-dire des régulations de l'action écrit Janet [19], les sentiments en accompagnent chacun des instants et stades.

\section{Nature et rôle des sentiments}

Il peut s'agir de régulations simples, comme les réactions, et complexes comme les jugements, ce que Janet nomme les actes secondaires [20] ; les émotions étant, elles, plutôt des troubles lorsque les sentiments sont mis en difficulté. Ces sentiments dont parle Janet sont au nombre de quatre pour les plus importants, comme l'observe également Charles Blondel [21], à savoir les sentiments d'effort, de fatigue, de tristesse, de joie [22], tous aujourd'hui confondus avec les émotions alors que celles-ci en expriment plutôt les troubles pour Janet. Le déclenchement de ces sentiments, comme leurs excès à l'annonce d'une action à faire (les euphories, les agitations sans but) et défauts lorsque l'on se sent inopérant, voire inutile (par exemple les sentiments du vide, les mélancolies, les inactions moroses), n'est alors pas perçu par lui comme séparé de chaque acte de vie, mais enveloppe en quelque sorte celui-ci comme s'ils le scandaient dans chacun de leurs intervalles, allant du souhait d'action à sa réalisation ; les excès et défauts étant contrôlés en moyenne par la synthèse d'ensemble qui constitue la personnalité, sauf quand la crainte de l'action est telle qu'ils s'autonomisent comme subconscience, voire se rendent même indépendants comme inconscience; nous avons déjà vu la distinction nécessaire à adopter entre ces deux termes.

\section{Névrose, comme peur de l'action et arrêt dans l'évolution}

L'action et sa signification montrent à l'organisme que chaque acte participe à son développement. Ainsi, (se) percevoir, penser, sentir (la sensation étant, elle aussi, une opération complexe et non pas seulement élémentaire pour Janet [23]), et ce, surtout de manière nouvelle, n'est pas sans risques lorsque tendanciellement est préférée la conservation plutôt que l'évolution. Cette inclination en direction de la seule conservation s'inscrit d'ailleurs au sein de la définition janétienne de la névrose lorsque la conservation ne signifie plus permanence de l'action mais sa crispation et son immobilisme dans les mêmes gestes et habitudes, Janet la décrivant précisément comme étant un arrêt dans la formation des fonctions psychiques [24].

Pour l'observer, Janet indique le lien entre la formation de la personnalité et le sentiment de pression [25] ou tension 
(elle est aussi simple et complexe [26]), lui-même coextensif au sentiment de l'effort, que Janet articule avec cet aspect particulier du sentiment de joie, l'élation ou sentiment d'enthousiasme (bien plus parlant que l'excitation trop vague et surtout d'ordre seulement physiologique [27]).

Cette pression est en effet parfois telle que l'on se trouve paradoxalement en incapacité à la fois de renoncer et de l'accomplir, ce qui suscite une émotion, c'est-à-dire, pour Janet, une interrogation d'ensemble sur ses propres capacités à agir [28].

Précisons ce point. La notion de personnalité décrit évidemment un processus; elle ne sort pas tout armée telle Athéna de la cuisse de Zeus. En son sein s'articulent des tendances, c'est-à-dire des « dispositions à certains actes » [29]. Elles viennent s'inscrire, selon divers niveaux ou stades élémentaires médians et supérieurs, comme des traits ou caractères de l'individu [30]. Ces tendances ont des intentions qui viennent prendre forme réelle en se détachant des rêveries [31] propres au personnage [32] pour devenir conscience [33] dans l'unité d'un moi réfléchissant quant à son rôle social [34], d'où le terme bien connu de personne (au sens durkheimien y compris, que cite d'ailleurs Janet [35]), ce masque, que le processus va imprimer d'une marque de fabrique singulière, celle de la personnalité affirmant une certaine composition. Ce qui ne va évidemment pas sans obstacles. D'où parfois un sentiment de lassitude et son trouble d'émotion, d'épuisement, quelquefois avant même d'agir ou une crainte d'accomplir, voire, à l'inverse, une euphorie dans l'ardeur dont l'extrême émotionnel atteint cependant le même résultat puisqu'elle peut également rendre impuissant par trop d'effervescence.

\section{Distinction entre émotion et sentiment}

Janet ne définissait pas l'émotion comme cette signification exprimant de la sensibilité, ce qu'il est coutume de faire de nos jours au détriment de la notion de sentiment réduite à son aspect intellectuel, Janet définissait l'émotion plutôt comme un trouble de l'action dont l'exagération peut paralyser... C'est que Janet ne limitait pas l'existence d'écueils au cœur de l'action à la seule interaction conflictuelle entre action et culpabilité comme le pense le freudisme [36], en particulier dans sa version lacanienne [37]. Janet mettait plutôt en avant un lien indissociable entre action-effort-personnalité et, en opposé, tout ce qui pouvait le désintégrer.

Et ce lien, si crucial de nos jours, dans un univers où celui-ci semble pourtant le découpler - il suffirait d'effleurer des touches et des écrans ou de cliquer, pour jouer, rencontrer, penser, devenir -, cette banalisation occulte le fait que l'effort, surtout dans sa dimension d'action efficace, semble de plus en plus être un vecteur de constitution et de différentiation de la personnalité, d'où la peur d'agir qui peut en découler.
D'ailleurs, et cela est valable pour toutes les époques, et sans doute toutes les sociétés, y compris les plus agressives, cette peur d'agir découle moins de la seule confrontation entre le désir et sa réalisation légitime, en un mot la culpabilité, que dans la capacité à réduire en nous l'écart [38] entre la décision d'agir et sa légitimité, ce qui implique de synthétiser l'effort, d'en hiérarchiser et d'en justifier les priorités comme le souligne Janet.

\section{Impossibilité de faire face au conflit au cour de l'impuissance d'action}

Ainsi, malgré l'interdit qui a, par ailleurs, d'autres sources que l'attachement familial (il provient également d'un référentiel sociétal - religieux, politique -, auquel l'on se trouve mentalement lié), une action, même antinormative, voire illégale, peut se réaliser, surtout dans l'anonymat urbain lorsque l'équilibre des tendances se trouve sauf. Le problème n'est alors pas tant le conflit avec la Loi que la possibilité de l'enfreindre. Par exemple, lorsqu'il s'agit d'une rupture avec tel groupe, voire dans le pas vu, pas pris de la transgression et son extrême criminel. Ehrenberg observe cette problématique chez Lacan qui aurait précisément attiré l'attention sur le conflit au sein même de la Loi (le Surmoi freudien) entre le désir d'être en quelque sorte son propre référent et le fait de se heurter à la figure du père pouvant y faire obstacle ou la peur de la castration [39]. Sauf que l'on en reste encore à la question de la seule culpabilité à laquelle ne peut être réduite la constitution de l'effort visant à l'action.

\section{Le problème n'est pas tant la limite de l'action que son impuissance à être}

Ehrenberg semble être, sur ce point, très paradoxal puisqu'après avoir énoncé que l'analyse de Janet sur la fatigue était bien plus opérante que celle de Freud sur la culpabilité - parce que Janet insiste bien plus sur la notion de synthèse que celle de conflit, la personnalité outrepassant en moyenne celui-ci lorsqu'elle s'unifie dans l'action [40] (sauf lorsque celui-ci est trop fort il peut la paralyser, voire en rétrécir l'amplitude) -, Ehrenberg amoindrit son constat en avançant ensuite [40] que c'est Freud qui aurait le mieux dessiné la préoccupation majeure du sujet moderne, à savoir, le lien conflictuel entre animalité (instincts du ça) et civilisation (Surmoi). Sauf que le problème n'est pas là, en tout cas pas pour Janet (qui, lui, parle plutôt de tendances que d'instincts [41]), puisqu'il ne s'agit pas tant de se demander seulement ce qui limite la capacité à agir, mais, aussi et surtout (car il y a là un primat dans les facteurs), ce qui l'empêche déjà de se constituer. À moins d'expliquer que l'interdit émerge en même temps que le désir ; ce qui voudrait dire que c'est la loi qui constitue l'action; plus encore que l'action n'est que la loi en acte ou encore que le 
désir émergerait uniquement pour voir l'interdit surgir ; ce qui est, là, confondre les niveaux, par exemple, la condition et le motif du but. Ehrenberg ne peut pas ne pas remarquer que lorsqu'il cite Lacan, celui-ci ne se préoccupe plus de savoir s'il y a conflit entre l'animalité et la loi (jusqu'à faire disparaître le premier terme, souligne Ehrenberg [42]), mais si l'action désirée peut persévérer...

De son côté, Janet ne met pas en opposition conflit et force, par exemple, celle qui constitue l'effort ; il cherche plutôt à comprendre comment elle se constitue et ce qui l'entrave. C'est là le lieu même de toute son œuvre, semble-t-il.

\section{Conflit et force, les deux faces d'une même médaille : celle de la synthèse d'action}

Il s'agit de définir, d'une part, comment la personnalité s'édifie et, d'autre part, ce qui fait qu'elle se délite. Il n'y a donc pas à percevoir d'un côté le conflit et ses divers aspects, qu'ils soient affectifs et sociaux, ou le positionnement de l'individu vis-à-vis de ces cercles d'action que sont la famille, les proches et le système institutionnel, le tout historiquement situé, et, de l'autre côté, la constitution du moi au moment de l'effort.

Les deux sont intriqués, et, précisément, la force d'une personne consiste en ce que l'individu dépasse le stade du personnage pour atteindre celui de la personnalité [43], à savoir ce moment où le moi s'accomplit en (se) réalisant dans le monde. Ce qui ne va pas évidemment sans conflit... Que celui-ci ait une forme plus ou moins collective, comme l'énonce Ehrenberg dans le même ouvrage [44], en un mot, qu'il semble avoir aujourd'hui plus de traits individualistes que dans les deux siècles précédents, ce qui impliquerait, par exemple, que la névrose psychasthénique ou dépressive liée à l'affaiblissement de la force d'action supplanterait dorénavant la névrose hystérique liée à une crise de délimitation et de détermination (dissociation et dédoublement) du moi par rapport à son positionnement vis-à-vis des divers cercles d'action, cette observation, quand bien même serait-elle exacte (ce qui est loin d'être évident, surtout dans sa linéarité historique), n'évacuerait pas le fait que l'on ne peut pas découpler ainsi conflit et constitution de l'action par lesquels le moi s'édifie. Janet établissait bien au contraire un lien entre, d'une part, ce qu'il nommait les tendances ou stades psychologiques et, d'autre part, leur affaiblissement fonctionnel, voire l'arrêt de leur évolution, c'est-à-dire, on l'a déjà énoncé, un des traits particuliers de la névrose :

Si on veut bien entendre par ce mot «évolution » ce fait qu'un être vivant se transforme continuellement pour s'adapter à des circonstances nouvelles, qu'il est sans cesse en voie de développement et de perfectionnement, les névroses sont des troubles ou des arrêts dans l'évolution des fonctions [45].

Puis il ajoute :

En rattachant les névroses au développement individuel et social, qui est si peu connu, on donne suffisamment satisfaction à ce sentiment d'étonnement qui poussait les premiers auteurs à considérer les névroses comme extraordinaires. En parlant des parties les plus élevées de chaque fonction, de celles qui sont encore en transformation, on sous-entend qu'il s'agit de phénomènes siégeant surtout dans le système nerveux, car c'est dans le système nerveux que s'élaborent et se perfectionnent les fonctions nouvelles des êtres vivants [46].

Ehrenberg admet cette qualification, celle de l'affaiblissement des fonctions [47], même s'il n'insiste pas assez sur leur aspect évolutif, et qu'il oublie de les lier avec la construction même du moi. Or, c'est cela même qui préoccupe Janet. Ainsi, lorsqu'il expose les divers moments de la formation de la personnalité, à savoir lorsque l'on découpe dans le jet des interactions un objet puis qu'on l'utilise, ensuite qu'on le distingue d'autres objets jusqu'à l'expérimenter et, de là, à en affiner l'interaction qui nous lie à lui afin de renforcer cette liberté qui permet au moi de basculer du personnage à la personnalité, de l'imaginaire narcissique au rôle social propre, au reliable man dont parle Janet à plusieurs reprises.

C'est ce qu'il faut entendre, semble-t-il, par cette idée d'évolution des fonctions.

Janet établit, en effet, un lien entre stades de l'intelligence et stades psychologiques dont l'arrêt dans l'évolution forme névrose et par là affaiblissement. Janet délimite ainsi l'aspect proprement psychologique et sa variante pathologique qui tournent tous deux autour de la notion d'effort.

\section{Rôle de l'effort dans l'organisation du Réel}

L'effort va en effet hiérarchiser les priorités, synthétiser leurs jeux de force, c'est-à-dire permettre de créer tout d'abord un objet, être ou espace-temps immédiat (l'asséritif dit Janet [48]) dont l'absolu se perçoit par exemple dans la «pensée symbolique » lorsque «des images, des symboles » sont traités comme des « êtres » [49] remarque Janet, tandis que son excès va s'exprimer dans ce qu'il nomme la « croyance pithiatique » [50] à savoir le fait qu'il n'y ait aucun « contrôle ». L'effort va ainsi cristalliser la tension en attention volontaire [51], mais s'il s'agit d'entrer dans le monde, de nouer contact avec autrui, il faut chercher à lui donner un visage avec du sens doué de réalité, c'est le stade réfléchi ajoute-t-il :

L'être, c'est ce que l'on croit par assentiment immédiat, le réel c'est ce que l'on croit après réflexion [52]. 
Janet fait d'ailleurs remarquer cela à ce propos :

Il est curieux de remarquer que les troubles $d u$ sentiment $d u$ réel, la perte $d u$ réel, les recherches angoissantes $d u$ réel, troubles si fréquents et si intéressants dans les névroses, se présentent toujours chez des individus qui ont des troubles de la croyance réfléchie [53].

Janet précise que cette perte se double d'une disparition des jugements que sont pour lui les sentiments et qu'il nomme « actes secondaires » [54], c'est-à-dire ces actes qui viennent moduler et scander nos moindres gestes et pensées en leur donnant ou en leur retirant du sens par des jugements qui viennent ainsi réguler l'action déclenchée.

Or, lorsque l'on ne croit [55] plus à ses capacités d'action et de réflexion, le fait d'émettre une pensée qui va donner un avis sur le déroulement des faits et gestes semble impromptue ; aussi Janet observe une accentuation du silence [56], une sorte de rétrécissement de l'esprit [57], doublée d'irritation et de fatigue. Nous le vivons, à des degrés moindres, tous les jours. Janet complexifie encore plus la notion d'effort lorsqu'il indique par ailleurs d'autres stades [58] qui approfondissent les stades précédents de l'immédiateté et du réflexif, comme ceux du stade rationnel lorsque «l'esprit est devenu capable de donner de la force à des formules logiques ou morales et de les transformer en ordres puissants » [59] ; ce qui implique ensuite pour Janet un degré de plus, celui du stade expérimental, c'est-àdire de cette « grande sincérité, non seulement avec les autres, mais avec soi-même » [60], puisqu'il s'agit d'observer le degré de vérité de toutes ces formules, que le stade dit progressif ne peut par la suite qu'affiner :

Les philosophes ont eu très souvent des discussions interminables à propos de la valeur de tel ou tel procédé d'explication, par la finalité ou par le déterminisme. C'est qu'ils ont toujours négligé de tenir compte des stades psychologiques auxquels ils se plaçaient. L'explication, comme les lois de la raison, comme la conduite, change de nature suivant le degré de l'évolution psychologique : il ne faut jamais se figurer qu'une explication gardera la même forme et sera valable pour tous les hommes. Il faut expliquer autrement à un débile mental au niveau asséritif qu'à un savant de nos jours au niveau expérimental, et il ne faut jamais oublier qu'un jour on rira de notre physique mathématique comme nous nous moquons des syllogismes et des forces occultes du moyen âge [61].

Dans ce même registre stipulant l'existence de stades et de leur progressivité psychologique, notons brièvement que Janet avait réfléchi sur la façon dont un Lévy-Bruhl classait, au début du $\mathrm{xx}^{\mathrm{e}}$ siècle, les sociétés en prélogiques et logiques [62], non pas par historisme comme le croit Raymond Boudon [63], mais parce que Lévy-Bruhl cherchait à classer en sociétés qui ne mettent pas en avant le principe de noncontradiction et celles qui le font. Autrement dit, il y aurait une différence à effectuer entre les sociétés qui n'opèreraient pas de distinction entre imaginaire et réel, entre spirituel et matérialité et celles qui le réaliserait ; tout en ne séparant cependant pas totalement ces deux domaines, puisque les champs du religieux et de l'art expriment toujours cette présence de l'imaginaire et du spirituel. Janet en avait néanmoins conclu que cette distinction entre prélogique et logique n'était cependant pas assez précise parce que l'absence des stades expérimentaux et progressifs dans les sociétés dites prélogiques, c'est-à-dire l'inexistence de pratiques vérifiant les présupposés de l'action, ne signifiait pas pour autant que ces populations n'étaient pas dotées des stades réfléchis et rationnels au sens large de construire une intention et de donner une signification à l'action déduite. Autrement dit encore, les prémisses permettant la constitution d'une évaluation étaient présentes, même si elles n'étaient pas aussi abouties que dans les sociétés ayant privilégié les stades expérimentaux et progressifs, ce que l'on nomme aujourd'hui la sécularisation de la société moderne et sa technicisation. Par ailleurs, Janet récusait l'idée que l'existence de tels stades implique nécessairement qu'ils agiraient en constance, via le principe de non-contradiction, au sein de nos pratiques de vie ; il n'en tirait pas du tout cette conclusion, contrairement à certaines affirmations, on l'a vu [64]. Notre chair indique, par exemple, une conduite de l'effort marquée par des concentrations de tension [65] et d'attention volontaire [66] qui donnent un sens à nos faits et gestes en chaque instant. Mais, aussi, le comportement peut, comme on le sait de mieux en mieux, impliquer une conduite d'affaiblissement, qui se traduit par des peurs de l'action, des distractions accentuées jusqu'à nous empêcher de voir ce qui est parfois sous nos yeux, ou d'agir au moment où il le faut.

De tels aspects ont été étudiés, en premier, et de façon la plus convaincante, me semble-t-il, par Pierre Janet. D'où la nécessité, je l'ai déjà formulé, de nous en imprégner pour cerner les problèmes d'aujourd'hui à l'époque biotechnologique, techno-urbaine, communicationnelle et cognitive, où l'effort et son imaginaire s'accomplissent beaucoup plus mentalement et se trouvent d'autant plus fragilisés.

\section{Différence entre Freud et Janet dans la pratique scientifique}

\section{Contextualité historique}

Freud écrit cela dans Ma vie et la psychanalyse :

Je lui proposai (à Breuer) une publication faite en commun, idée contre laquelle il commença par se défendre violemment. Il finit par céder, après qu'entre-temps les travaux de Janet eussent anticipé sur une partie de ses résultats : le rattachement des symptômes hystériques à des impressions de la vie et leur levée de par leur reproduction sous hypnose in statu nascendi. Nous fimes paraître, en 
1893, une étude préalable : «Du Mécanisme psychique des phénomènes hystériques ». En 1895 suivit notre livre:

Études sur l'hystérie [67].

Dans ces conditions, ne faut-il pas s'étonner que Janet, en 1913, puisse citer ce qu'il écrivait, en 1893, lors de la parution de L'État mental des hystériques?

Nous sommes heureux que MM Breuer et Freud aient vérifié récemment notre interprétation déjà ancienne des idées fixes chez les hystériques [68].

Car, entre 1885 et 1888 , soit plusieurs années avant Freud, Janet avait déjà publié toute une série d'articles préparant ces deux ouvrages principaux ultérieurs (L'automatisme... et L'État mental...), dans la Revue philosophique de Ribot (1885) et dans la revue de la Société de Psychologie Physiologique de Charcot (1886) [69]. Ces articles avaient pour trait la catalepsie, le somnambulisme, la suggestion, le rêve, l'habitude, la passion, etc. Notons, par exemple en 1886, l'article intitulé « Les actes inconscients et le dédoublement de la personnalité pendant le somnambulisme provoqué », publié dans la Revue Philosophique de la France et de l'Étranger [70]. De 1886 à 1891, Freud relate de son côté, «j’ai peu travaillé scientifiquement et n'ai presque rien publié » [71]. Attardons-nous encore sur cette époque.

\section{Quelques exemples d'études cliniques faites par Janet qui le différencie de Freud}

Janet peut, par exemple, faire état de ses travaux dans les divers articles cités plus haut parce qu'il s'appuie sur des faits précis, des cas cliniques en particulier traumatiques, dont l'un, celui de Marie, a été repris entièrement [72] par Henri F. Ellenberger pour illustrer l'œuvre de Pierre Janet dans sa monumentale étude Histoire de la découverte de l'inconscient.

Voilà comment Janet présentait ce cas :

Cette jeune fille fut amenée de la campagne à l'hôpital du Havre à l'âge de 19 ans, parce qu'on la considérait comme folle et que l'on désespérait presque de sa guérison. En réalité, elle avait des périodes de crises convulsives et de délires qui duraient des journées entières. Après quelque temps d'observation, il était facile de constater que la maladie se composait d'accidents périodiques revenant régulièrement au moment de ses époques et d'autres accidents moins graves se prolongeant et survenant irrégulièrement dans les intervalles.

Considérons d'abord les premiers. À l'approche de ses règles, Marie changeait de caractère, devenait sombre et violente, ce qui ne lui était pas habituel, et avait des douleurs et des secousses dans tous les membres. Cependant, les choses se passaient à peu près régulièrement pendant la première journée mais, 20 heures à peine après le début, les règles s'arrêtaient subitement et un grand frisson secouait tout le corps, puis une douleur vive remontait lentement du ventre à la gorge, et les grandes crises d'hystérie commençaient. Les convulsions, quoique très violentes, ne duraient pas longtemps et n'avaient jamais l'aspect de tremblements épileptö̈des : mais elles étaient remplacées par un délire des plus longs et des plus forts. Tantôt elle poussait des cris de terreur, parlant sans cesse de sang et d'incendie et fuyant pour échapper aux flammes, tantôt elle jouait comme un enfant, parlait à sa mère, grimpait sur le poêle ou sur les meubles et dérangeait tout dans la salle. Ce délire et ces convulsions alternaient, avec d'assez courts instants de répit, pendant 48 heures. La scène se terminait par plusieurs vomissements de sang après lesquels tout rentrait à peu près dans l'ordre. Après une ou deux journées de repos, Marie se calmait et ne se souvenait de rien. Dans l'intervalle de ces grands accidents mensuels, elle conservait de petites contractures tantôt aux bras ou à la poitrine, dans les muscles intercostaux, des anesthésies variées et très changeantes et surtout une cécité absolue et continuelle de l'œil gauche (...). En outre, elle avait de temps en temps de petites crises sans grand délire, mais qui étaient caractérisées surtout par des poses de terreur. Cette maladie, rattachée si évidemment aux époques menstruelles, semblait uniquement physique et peu intéressante pour le psychologue. Aussi ne me suis-je d'abord que fort peu occupé de cette personne. Tout au plus ai-je fait avec elle quelques expériences d'hypnotisme et quelques études sur son anesthésie, mais j'évitai tout ce qui aurait pu la troubler vers l'époque où approchaient les grands accidents. Elle resta ainsi sept mois à l'hôpital sans que les diverses médications et l'hydrothérapie qui furent essayées eussent amené la moindre modification. D’ailleurs, les suggestions thérapeutiques, en particulier les suggestions relatives aux règles, n'avaient que de mauvais effets et augmentaient le délire.

Vers la fin du huitième mois, elle se plaignait de son triste sort et disait avec une sorte de désespoir qu'elle sentait bien que tout allait recommencer : "Voyons, lui disje par curiosité, explique-moi une fois ce qui se passe quand tu vas être malade. - Mais vous le savez bien,... tout s'arrête, j'ai un grand frisson et je ne sais plus ce qui arrive ». Je voulus avoir des renseignements précis sur la façon dont ses époques avaient commencé et comment elles avaient été interrompues. Elle ne répondit pas clairement, car elle paraissait avoir oublié une grande partie des choses qu'on lui demandait. Je songeai alors à la mettre dans un somnambulisme profond, capable, comme on l'a $v u$, de ramener des souvenirs en apparence oubliés, et je pus ainsi retrouver la mémoire exacte d'une scène qui n'avait jamais été connue que très incomplètement. À l'âge de 13 ans, elle avait été réglée pour la première fois, mais par suite d'une idée enfantine et d'un propos entendu et mal compris, elle se mit en tête qu'il y avait à cela quelque 
honte et chercha le moyen d'arrêter l'écoulement le plus tôt possible. Vingt heures à peu près après le début, elle sortit en cachette et alla se plonger dans un grand baquet d'eau froide. Le succès fut complet, les règles furent arrêtées subitement, et, malgré un grand frisson qui survint, elle put rentrer chez elle. Elle fut malade assez longtemps et eut plusieurs jours de délire. Cependant, tout se calma et les menstrues ne reparurent plus pendant cinq ans. Quand elles ont réapparu, elles ont amené les troubles que j'ai observés. Or, si l'on compare l'arrêt subit, le frisson, les douleurs qu'elle décrit aujourd'hui en état de veille avec le récit qu'elle fait en somnambulisme et qui, d'ailleurs, a été confirmé indirectement, on arrive à cette conclusion : tous les mois, la scène du bain froid se répète, avec le même arrêt des règles et un délire qui est, il est vrai, beaucoup plus fort qu'autrefois, jusqu'à ce qu'une hémorragie supplémentaire ait lieu par l'estomac. Mais, dans sa conscience normale, elle ne sait rien de tout cela et ne comprend même pas que le frisson est amené par l'hallucination du froid; il est donc vraisemblable que cette scène se passe au-dessous de cette conscience et amène tous les autres troubles par contrecoup.

Cette supposition vraie ou fausse étant faite, et après avoir pris l'avis du Dr Powilewicz, j'ai essayé d'enlever de la conscience somnambulique cette idée fixe et absurde que les règles s'arrêtaient par un bain froid. Je ne pus tout d'abord y parvenir; l'idée fixe persista, et l'époque menstruelle qui arrivait deux jours après fut à peu près comme les précédentes. Mais, disposant alors de plus de temps, je recommençai ma tentative : je ne pus réussir à effacer cette idée que par un singulier moyen. Il fallut la ramener par suggestion à l'âge de 13 ans, la remettre dans les conditions initiales du délire, et alors la convaincre que les règles avaient duré trois jours et n'avaient été interrompues par aucun accident fâcheux. Eh bien, cela fait, l'époque suivante arriva à sa date et se prolongea pendant trois jours, sans amener aucune souffrance, aucune convulsion, ni aucun délire.

Après avoir constaté ce résultat, il fallait étudier les autres accidents. Je passe sur des détails de la recherche psychologique qui fut quelquefois difficile : les crises de terreur étaient la répétition d'une émotion que cette jeune fille avait éprouvée en voyant, quand elle avait 16 ans, une vieille femme se tuer en tombant d'un escalier; le sang dont elle parlait toujours dans ses crises était un souvenir de cette scène; quant à l'image de l'incendie, elle survenait probablement par associations d'idées, car elle ne se rattache à rien de précis. Par le même procédé que tout à l'heure, en ramenant le sujet par suggestion à l'instant de l'accident, je parvins, non sans peine, à changer l'image, à lui montrer que la vieille avait trébuché et ne s'était pas tuée, et à effacer la conviction terrifiante : les crises de terreur ne se reproduisent plus.
Enfin, je voulais étudier la cécité de l'œil gauche, mais Marie s'y opposait lorsqu'elle était éveillée, en disant qu'elle était ainsi depuis sa naissance. Il fut facile de vérifier, au moyen du somnambulisme, qu'elle se trompait : si on la change en petit enfant de cinq ans suivant les procédés connus, elle reprend la sensibilité qu'elle avait à cet âge et l'on constate qu'elle y voit alors très bien des deux yeux. C'est donc à l'âge de six ans que la cécité a commencé. À quelle occasion? Marie persiste à dire, quand elle est éveillée, qu'elle n'en sait rien. Pendant le somnambulisme et grâce à des transformations successives pendant lesquelles je lui fais jouer les scènes principales de sa vie à cette époque, je constate que la cécité commence à un certain moment à propos d'un incident futile. On l'avait forcée, malgré ses cris, à coucher avec un enfant de son âge qui avait de la gourme sur tout le côté gauche de la face. Marie eut, quelque temps après, des plaques de gourme qui paraissaient à peu près identiques et qui siégeaient à la même place ; ces plaques réapparurent plusieurs années à la même époque, puis guérirent, mais on ne fit pas attention qu'à partir de ce moment, elle est anesthésique de la face du côté gauche et aveugle de l'œil gauche. Depuis, elle a toujours conservé cette anesthésie, du moins, pour ne pas dépasser ce qui a pu être observé, à quelque époque postérieure que je la transporte par suggestion, elle a toujours cette même suggestion, elle a toujours cette même anesthésie, quoique le reste du corps reprenne à certaines époques sa sensibilité complète. Même tentative que précédemment pour la guérison. Je la ramène avec l'enfant dont elle a horreur, je lui fais croire que l'enfant est très gentil et n'a pas la gourme, elle n'est qu'à demi convaincue. Après deux répétitions de la scène, j’obtiens gain de cause et elle caresse sans crainte l'enfant imaginaire. La sensibilité du côté gauche réapparaît sans difficulté et, quand je la réveille, Marie voit clair de l'œil gauche.

Voilà cinq mois que ces expériences ont été faites, Marie n'a plus présenté le plus léger signe d'hystérie, elle se porte fort bien et surtout se renforcit beaucoup. Son aspect physique a absolument changé. Je n'attache pas à cette guérison plus d'importance qu'elle n'en mérite, et je ne sais pas combien de temps elle durera, mais j'ai trouvé cette histoire intéressante pour montrer l'importance des idées fixes subconscientes et le rôle qu'elles jouent dans certaines maladies physiques aussi bien que dans les maladies morales.

Le cas de Nelly fut, lui, présenté par Janet, en 1913, en pleine polémique avec Freud sans doute afin de bien signifier que contrairement aux allégations propagées par les freudiens, les problèmes aux apparences sexuelles ne lui étaient pas étrangers [73] :

Une femme de 30 ans, Newy, récemment mariée et déjà enceinte, est toujours restée complètement indifférente aux caresses de son mari. "Mon mari n'est pas pour moi ce qu'il devrait être, répète-t-elle, c'est là ce qui me rend si 
malade: je ne sens rien quand je suis près de lui, il y a un vide entre nous deux. Il n'arrive pas à faire que je puisse l'aimer... Moi qui avais tellement le désir de me marier, je n'éprouve rien dans le mariage et j'ai maintenant l'idée de quitter mon mari, de me sauver n'importe où... Cela m'énerve de ne sentir rien, d'être comme un morceau de bois, et c'est là ce qui me rend malade».

Remarquons d'abord que le trouble est plus général que ne le pense la malade. Beaucoup d'autres sentiments sont également troublés, notons seulement le trouble curieux du sentiment de propriété : "Rien n'est à moi dans cet appartement... Je ne suis chez moi dans aucune de ces chambres, elles me semblent étrangères et comme mortes. Ces costumes, ces robes que l'on m'a achetés depuis le début des fiançailles ne sont pas à moi... Si je l'osais, je rechercherai mes vieilles robes et je les mettrais, car celleslà sont à moi... Si j'essaye aujourd'hui d'acheter quelque chose pour le ménage, je ne pourrai même pas en rentrant défaire le paquet, car ce qui est dedans ne sera pas à moi et ne m'intéressera pas. On peut venir prendre tout ce qui est chez moi, je ne retiendrai rien, je suis attachée à aucun objet comme à aucune personne ». Non seulement tous les sentiments, mais également tous les actes sont troublés: elle ne peut absolument rien faire : "Je n'ai pas l'idée de m'installer ici, de mettre en train ce petit ménage. Si j'essaye de commencer une action, je me sens épuisée d'avance et je me mets à pleurer sans rien faire; mes actions ne sont pas réelles, pas plus que tout le reste... je ne peux même pas me décider à dormir... ».

Ces troubles généraux sont antérieurs aux relations sexuelles avec le mari, ils ont existé gravement dès le début des fiançailles, ils existaient même antérieurement quoique moins accusés. C'est une femme d'une volonté extrêmement faible qui a toujours vécu auprès de sa mère et de sa søur et qui était conduite par elles dans toutes ses actions. «Ma mère et ma sœur décidaient tout pour moi et j'étais perdue, ahurie, si je me trouvais un moment sans elles ». Newy a déjà eu des crises de scrupules, moins fortes, il est vrai, que celleci, mais caractéristiques quand elle a été en vacance chez une tante loin de sa mère et de sa sæur.

Dans ces conditions, il me semble que ce serait une grave erreur que de placer les troubles génitaux à l'origine de tout cela et de lui faire avouer, ce qui est facile, quelques masturbations anciennes dont elle n'est que trop disposée à s'accuser.

Cette femme ne fait pas correctement l'acte génital de la même manière qu'elle ne peut pas correctement faire l'acquisition d'un objet ou commander son dîner, l'aboulie génitale n'est qu'une manifestation de son aboulie générale. Il s'agit d'une personne déjà déprimée depuis plusieurs années, dont la dépression tient à bien des causes, l'hérédité certainement, à l'éducation qui a été absurde, à une mauvaise hygiène physique et morale. Cette personne prédisposée a été épuisée par les difficultés des fiançailles, elle a été bouleversée en quittant sa mère et sa sæur par la nouveauté de la vie conjugale, de la vie à deux, par l'indépendance et la solitude où elle était laissée, par le changement d'appartement et, enfin, par les débuts de la grossesse. Cet épuisement, cet abaissement de la tension psychologique a troublé toutes les actions, et, en particulier, a rendu impossible les actes d'acquisition avec sentiment de propriété et l'acte génital avec jouissance, parce que ce sont précisément des phénomènes psychologiques de haute tension.

Ce qui prouve la justesse de notre interprétation, c'est que cette malade se rétablit peu à peu sans que l'on se préoccupe le moins du monde de modifier ou de mieux organiser ses relations sexuelles. Il a suffi de soins hygiéniques, d'une direction morale qui diminue la difficulté des décisions à prendre, d'une éducation graduelle de son initiative, et un jour, la malade est toute surprise de constater que ses meubles deviennent sa propriété : "la salle à manger est bien à moi, mais pas encore la chambre à coucher...». Quand un jour elle a réussi avec beaucoup d'aide à organiser un petit dîner chez elle, elle en est fière et remonte assez sa tension pour pouvoir aimer le mari, pour avoir une jouissance complète et pour pouvoir, ce qu'elle croyait impossible, dormir toute une nuit près de lui. Plus tard, les fonctions génitales oscillent exactement comme l'activité générale, elles baissent quand tous les autres actes baissent aussi, elles remontent et parviennent à l'acte complet quand il y a eu excitation de la volonté sans aucun traitement relatif aux fonctions sexuelles. Il en est de même chez beaucoup de malades : bien des observations recueillies sans parti pris et de simples expériences dirigées par la méthode d'induction nous montrent que des troubles sexuels en apparence très graves et des souvenirs traumatiques en rapport avec eux sont des phénomènes secondaires qui dépendent de la maladie elle-même, bien loin de pouvoir l'expliquer.

Quand j'ai exprimé autrefois des réflexions de ce genre sur le caractère secondaire des troubles sexuels dont parlait M. Freud, je me suis attiré la même critique sévère qui avait déjà frappé M.J.E. Donley et I.H. Coriat. Dans ses études sur la pathogénie de l'anxiété morbide [74], M.E. Jones rappelle les remarques que j'avais faites et sans les discuter; il les supprime par ce reproche capital: «M. Janet, dit-il, n'a pas fait la psychoanalyse de ses sujets..., s'il avait fait cette psychoanalyse, il aurait forcément constaté que ces défauts des fonctions génitales sont des troubles spécifiques dus aux premiers développements de la vie sexuelle des malades». Hélas! M. Jones a raison, je n'ai pas fait la psychoanalyse, c'est-à-dire que je n'ai pas interprété les dires des malades dans le sens d'un dogme arrêté d'avance et je ne pouvais pas le faire, justement parce que je ne croyais pas au dogme et que je cherchais à constater sa vérité (...). 
Cette importance accordée aux événements sexuels résulte logiquement, si je ne me trompe, du caractère des premières études de M. Freud. Comme nous l'avons vu, cet auteur a essayé de transformer d'une manière générale les conceptions de l'analyse psychologique sur les souvenirs traumatiques et sur la subconscience en les généralisant démesurément. Quand on est décidé à retrouver chez tous les névropathes un souvenir d'une aventure émotionnante, capable de bouleverser la conscience, quand on admet a priori que ce souvenir sera toujours plus ou moins refoulé, dissimulé sous des symboles et des métaphores et qu'il ne sera exposé par le malade qu'avec des réticences et des efforts, on arrive à peu près forcément à la découverte de secrets d'alcôve. Dans notre civilisation, les événements qui ont le plus souvent déterminé des émotions petites ou grandes, les faits dont les hommes et les femmes n'aiment pas d'ordinaire à parler librement, qu'ils expriment par des allusions, avec des mots latins qui bravent l'honnêteté, ce sont toujours les aventures de la vie sexuelle. La manière dont M. Freud avait compris le souvenir traumatique et les idées fixes subconscientes l'a conduit à donner cette grande importance à des aventures sexuelles racontées à demi-mot. Il ne faut pas être surpris s'il a apporté dans cette étude sa méthode d'interprétation ingénieuse et de généralisation hardie. Aussi croyons-nous qu'il faut résumer cette nouvelle étude de la même manière que les précédentes. L'analyse psychologique avait constaté à titre d'observation et d'hypothèse le rôle considérable de la sexualité dans les névroses ; la psychoanalyse a transformé cette notion et en a fait, si je puis emprunter un mot de M. Bleuler et de M. Ladame, le dogme de la pansexualité ».

\section{Mécompréhension de Janet par Freud}

Freud réagit violemment. Il l'explique dans Ma vie... [75], en intervenant rarement sur le fond, sauf en parlant de « résistance à l'analyse » rétorquant plutôt avec morgue et mépris « qu'il n'a jamais entendu parler de Janet lorsqu'il suivait l'enseignement de J.M Charcot à la salpêtrière en 1885 »; or, sur ce point, de détail certes, il semble bien que sa réaction émotionnelle n'aurait pas eu lieu d'être, au moins pour deux raisons :

- en premier lieu, Janet n'entre en contact direct avec Charcot via la salpêtrière qu'en 1886 ;

- en second lieu, la réaction de Janet est concevable lorsqu'il dit que Freud et Breuer se sont inspirés de sa recherche puisqu'il s'appuie pour le dire sur leurs seules publications effectives qui datent de 1896 (alors que luimême a publié plusieurs années auparavant sur ce point et s'est, précisément, fait connaître grâce à cela) et non pas sur l'idée qu'en serait fait dès 1880, Breuer qui en aurait... «parlé » à Freud... comme celui-ci l'explique dans sa biographie [76]. Ces choses-là ne se font pas ainsi dans la production scientifique.

En 1889, soit donc plusieurs années avant l'article Freud-Breuer de 1896, Janet rassemble plusieurs articles et publie sa thèse de philosophie sur L'automatisme psychologique où il démontre que l'hystérie a une base bien plus psychologique que physiologique ; il distingue également, quoique pas encore suffisamment, le terme de subconscient de celui d'inconscient pour bien spécifier que dans toute action psychologique la plus « anormale », une certaine forme de conscience reste perceptible [77].

\section{Charcot a plutôt travaillé avec Janet qu'avec Freud}

Charcot confie à Janet, en 1890, la direction d'un laboratoire de psychologie à la clinique de la Salpêtrière. Alors qu'en 1885, Charcot confie plutôt à Freud la traduction en allemand de ses « Nouvelles leçons » [78]... Il y a bien là une différence de niveau. Pourtant, il n'en faut pas plus pour que Freud écrivit dans Ma vie... que «Charcot m'agrée, m'introduisit dans son intimité et depuis lors j'eus ma pleine part de tout ce qui avait lieu à la clinique » [79]. Notons en passant, s'agissant de cette « pleine part », que lorsque Freud propose à Charcot de diriger une thèse « ayant pour but la comparaison entre les paralysies hystériques et les organiques » [80], Charcot décline l'offre, ce qui fait dire à Freud que Charcot «était d'accord avec moi, mais on pouvait aisément voir qu'au fond il n'avait aucune prédilection pour une étude psychologique approfondie de la névrose » [81]. Or, Charcot n'a eu de cesse d'étudier l'hystérie dans ses fondements spécifiquement psychologiques, par exemple, son ouvrage de 1884 intitulé Pour guérir les paralysies psychiques par des procédés psychologiques. Sauf que Charcot ne devait sans doute pas partager l'avis de Freud stipulant que toute névrose aurait pour base un refoulement sexuel. Janet reprit au fond la même position.

\section{Raison du succès mondain freudien}

Certes, Freud eut plus de succès, du moins au sein du grand public, que Janet, particulièrement avec la vogue du surréalisme et son engouement pour l'écriture automatique, du freudo-marxisme, et ensuite du développement personnel, jusqu'à refluer cependant considérablement aujourd'hui avec les polémiques attisées par la parution du Livre noir de la psychanalyse.

Le succès de Freud vient probablement du fait qu'il posait à la base de tout trouble sa consonance sexuelle lorsqu'elle s'avère conflictuelle et donc refoulée par la culpabilité, ce qui n'était pas pour déplaire à la fin du XIX ${ }^{\mathrm{e}}$ siècle, à l'heure de l'individualisme et de la liberté des mœurs en pleine explosion. Par ailleurs, il attirait l'attention 
sur le rôle des parents, ce qui semble être là son point le plus fort, quoique pas si inédit que cela à vrai dire, surtout si l'on observe que Freud posait comme trauma majeur la question de l'inceste alors que la figure du père ou de la mère joue bien plus le rôle de pattern de socialisation que rempart contre la pulsion d'accouplement [82].

\section{Janet s'oppose au monisme freudien sur l'origine des traumatismes}

Pour Janet, cette systématique ou pansexualisme, ne correspondait pas à ce qu'il étudiait, non pas parce que la dérivation sexuelle n'existait pas, mais parce que ce n'était pas son refoulement qui importait seulement, plutôt ce qu'il indiquait comme symptôme de quelque chose de plus profond encore : l'impuissance à agir. Ce qui incitait à se servir du sexe, comme de la nourriture (anorexie, boulimie), mais aussi de l'aboulie, de l'irascibilité, de la paralysie partielle ou hémiplégique, comme solutions compensatrices.

Janet ne partait donc pas de l'unicausalité castratrice présupposant que l'impossibilité d'atteindre le but sexuellement convoité créé le trouble. Mais plutôt ou à l'inverse, que c'est l'impossibilité même d'atteindre le but convoité en vue d'avoir éventuellement une activité sexuelle avec lui qui suscite le traumatisme.

Cette impossibilité peut avoir pour cause un sentiment d'infériorité comme le pensait Adler, mais pas nécessairement lié à la taille du pénis, plutôt dépendant du fait que les parents n'aient pas suffisamment accompagné l'enfant en reconnaissant sa capacité à agir, par exemple, jusqu'à devenir cause et à prendre plaisir. Cette donnée s'articule vraisemblablement à d'autres facteurs, sociaux, entre autres.

En tout cas, au niveau du symptôme, et lorsqu'aucune lésion n'était décelable, cette impossibilité se traduisait, par exemple, par un rétrécissement de la conscience, d'où découlait par ailleurs l'hystérie ou encore par un affaiblissement de la synthèse, qui permet de nous organiser en hiérarchisant les priorités et les croyances, mais ce qui déclenche ce rétrécissement et cette faiblesse, en un mot, la cause de l'impuissance à agir, pouvait être multiple, le trouble sexuel en étant pour ainsi dire l'une des conséquences.

\section{Janet refuse la polémique avec Freud}

Quoi qu'il en soit, Janet ne cherchait pas nécessairement à entretenir la polémique. Car son objet était d'avancer scientifiquement.

Il pouvait, par exemple, spécifier cela en 1913 à propos de Breuer et Freud (je soulignerai quelques mots significatifs) :

Ces auteurs montraient par des exemples très heureusement choisis que certains troubles étaient la conséquence de "réminiscences traumatiques» et leurs observations, je le constatais avec plaisir, étaient tout à fait analogues aux miennes. Tout au plus, ces auteurs changeaient-ils quelques mots dans leur description psychologique, ils appelaient psychoanalyse ce que j'appelais analyse psychologique, ils nommaient «complexus » ce que j'avais nommé "système psychologique » pour désigner cet ensemble de phénomènes psychologiques et de mouvements, soit des membres, soit des viscères, qui restait associé pour constituer le souvenir traumatique ; ils baptisaient du nom de "catharsis»ce que je désignais comme une dissociation des idées fixes ou comme une désinfection morale [83].

Et dans ce même rapport de 1913, si décrié, il pouvait également reconnaître le mérite de Freud sur tel ou tel aspect, par exemple sur le refoulement [84] ou, plus tard, en 1926, sur le lien entre angoisse et « acte sexuel arrêté avant sa consommation » [85]. Il ne faut donc pas s'étonner, dans ces conditions, celles de la recherche de la vérité, pour paraphraser un auteur que Janet affectionnait (Malebranche), que celui-ci, de passage à Vienne, ait voulu rencontrer Freud... mais ce dernier lui claqua la porte au nez. Le ressentiment n'est pourtant pas la meilleure des solutions pour l'affirmation de soi disait Nietzsche...

\section{E. Roudinesco, quelques dizaines d'années après, poursuit la polémique en abaissant Janet}

En effet, «pourquoi tant de haine » pour Janet, non seulement de la part de Freud, mais aussi de nos jours, plusieurs décennies après, de la part d'une Elisabeth Roudinesco (en reprenant ainsi le titre dont elle affubla sa réponse à l'encontre du Livre noir...) ?

À lire les passages qu'elle consacre à Janet dans son Histoire de la psychologie en France, il n'est question que de jalousie envieuse vis-à-vis de Freud, facteur premier de sa critique envers la psychanalyse selon elle, et ce, à partir de travaux, sinon faux, du moins controversés et en fin de compte dépassés.

Elle parle de ce refus entêté qu'aurait la France en général, et Janet en particulier, de placer le refoulement sexuel au centre de l'analyse comme l'a fait Freud, et indique que Freud et Janet entendent «se poser comme unique héritier de l'enseignement du grand Charcot» [86] alors que nous avons montré qu'il n'en était rien : Charcot a bien vu la qualité précieuse d'un Janet en lui confiant la direction d'un laboratoire parce qu'il ne partage simplement pas l'unilatéralité causale de Freud. Par ailleurs, Roudinesco fait état, tout de go, d'une lettre de Jung où celui-ci relate son entrevue avec Janet en des termes peu amènes [87] ; or, on sait bien par la suite que Jung revint sur ce jugement abrupt et pencha franchement du côté de Janet... 


\section{Conceptions freudiennes et janetiennes de la névrose et de l'inconscient}

À la différence de la psychanalyse freudienne, l'analyse psychologique que Janet fonde, en 1885, ne base pas, on l'a dit, tous les troubles psychiques sur un traumatisme infantile sexuel refoulé (le désir impossible de l'inceste) comme le pense Sigmund Freud :

(...) le complexe d'Edipe peut être considéré comme le noyau des névroses [88].

Car ce qui est le noyau des névroses pour Janet ce n'est pas cette pulsion mécanique, énergétique, de fusion avec la mère, mais l'impossibilité d'évoluer, de grandir, de faire en un mot l'effort de (se) supporter (dans) son rapport au monde, bref, de croire en sa capacité à être ou la confiance en soi, dans tous les sens de ce dernier terme, d'où le rétrécissement de la conscience, la fatigue, le sentiment de vide. Ainsi, Janet avance, à la suite de Charcot - qui fut le premier à distinguer l'hystérie de l'épilepsie [89], c'est-à-dire à ne pas réduire systématiquement des troubles psychiques à des lésions physiologiques -, que c'est tout d'abord l'idée même du traumatisme ou choc qu'il s'agit de considérer car celui-ci, en surgissant, met en question la confiance en soi, c'est-à-dire la croyance en ses propres capacités à agir.

Janet, tout en visant à établir une psychologie générale qui permettrait de dégager le psychisme humain en tant que tel et non pas seulement dans son aspect pathologique, approfondit ainsi le travail de Charcot, même s'il s'écarte des hypothèses de travail de certains de ses élèves travaillant sur la métalloscopie, à savoir cette capacité qu'auraient certains métaux à faire du transfert [90], c'està-dire à déplacer magnétiquement certains troubles. Telle n'est pas la démarche de Janet [91] qui cherche l'origine proprement psychologique des troubles, en distinguant le subconscient - c'est-à-dire, nous l'avons montré, cette partie mise en retrait de la conscience qui n'est pas, pour le moment, au devant de la scène, s'en offusque et le manifeste par divers signes d'impatience -, de l'inconscient qui en est plutôt l'excès pathologique, c'est-à-dire la pointe tout à fait déréglée allant jusqu'à la paralysie. En 1913, Janet peut ainsi écrire, nous l'avons déjà cité :

Ce qui caractérise la subconscience, ce n'est pas que la tendance diminue ou reste latente, c'est au contraire que les tendances se développent, se réalisent fortement sans que les autres tendances de l'esprit soient averties de leur réalisation et sans qu'elles puissent travailler à s'y opposer [92].

Par « tendance », Janet entend, rappelons-le, des « dispositions à certains actes » [93] qu'il s'agit de mettre en accord pour l'action, donc de les hiérarchiser, parce que des tendances prennent le pas sur les autres, comme ici le fait d'écrire ces lignes au lieu d'aller se promener ou seulement d'en parler. Sauf que les tendances qui aspirent à cette dernière action peuvent se rebeller d'être ainsi mises en suspens ; elles peuvent susciter une fatigue, un ennui, obscurcir la compréhension de ce travail, s'affaiblir, rétrécir en un mot le champ de la conscience en butant sur une idée fixe, une obsession, une phobie, bref, refuser de progresser - c'est ce que Janet nomme d'ailleurs la névrose, on le verra plus loin - ou, au contraire, travailler en souterrain, et ce, positivement comme Janet l'écrit, en 1898, rappelons-le encore :

Il y aurait, je crois, toute une étude psychologique des plus curieuses à faire sur cette rêverie intérieure et continuelle qui joue chez beaucoup d'hommes un rôle considérable. On pourrait étudier le contenu de ces rêveries; on y verrait quelquefois de curieux travaux psychologiques qui s'effectuent en nous, à notre insu. C'est grâce à ce travail subconscient que nous trouvons tout résolus des problèmes que peu de temps auparavant nous ne comprenions pas [94].

L'acte inconscient proprement dit, c'est-à-dire dissociatif en ce qu'il anesthésie, paralyse plus en profondeur, n'est pas aussi joyeux.

\section{Progressivité théorique de Janet dans la distinction entre subconscient et inconscient}

À ce stade, on peut cependant préciser deux choses : en premier lieu, Janet ne fait certes pas une aussi nette distinction entre subconscient et inconscient dans ses premiers écrits ; par exemple, dans L'automatisme psychologique (1889), Janet parle plutôt de subconscience comme inconscience particulière, tout en réservant le terme de dissociation à son expression extrême. Néanmoins, remarquons qu'en 1898, soit neuf ans plus tard, Janet souligne l'aspect dynamique positif d'un subconscient. Et, en 1913, Janet précise sa pensée sur le subconscient en récusant son aspect latent ou faible. En second lieu, la conception janétienne se distingue de la conception freudienne en ce qu'il ne s'agit pas de focaliser ce travail non conscient sur une seule tendance, la tendance sexuelle qui, refoulée parce que n'étant pas admise à accomplir l'inceste, structurerait ensuite tout acte, qu'il soit suspensif comme le subconscient ou dissociatif comme l'inconscient. Pour Janet, la conscience reste toujours en jeu, même lorsque celle-ci se rétrécit ou s'affaiblit.

\section{Conscience, subconscience, inconscience chez Janet}

Ce supposé primat de l'inconscient sur la conscience dont se vante Roudinesco est en réalité aujourd'hui rejeté fortement par les neurosciences, alors que, pourtant, certains de ses chercheurs, comme Lionel Naccache, restent dans l'ensemble bien disposés à l'égard de Freud [95]. La conscience est plutôt, au contraire, si présente qu'elle 
implique pour l'analyse de ne pas se tromper dans l'interprétation, surtout lorsqu'il s'agit de rêves que Janet prenait en considération, mais n'en faisait pas l'alpha et l'oméga car l'on peut souvent se tromper ou plus exactement réagir bien trop logiquement alors que la réalité s'avère bien plus complexe, à l'instar des antinomies kantiennes qui sont toutes les quatre très logiques alors qu'elles sont fausses en ce sens qu'elles ont besoin d'une confrontation au réel, c'est-à-dire à ce qui ne se déduit pas de leur cohérence interne, mais implique une correspondance vérifiable en causalité.

Janet avait précisément l'ambition de cerner cette complexité, contradictoire, du psychisme humain en tant que tel et sa pathologie : l'individu est composé de tendances contradictoires, certaines s'affirment, d'autres sont écartées, comment ? Parce qu'il existe une volonté qui hiérarchise, à savoir la conscience, comme l'analyse plus amplement Janet, en 1926, dans De l'angoisse à l'extase.

Dans ces conditions, on peut comprendre pourquoi Janet distingue également le jeu subconscient-inconscient avec la conception plus générale de l'inconscient perçu depuis Leibniz comme des espèces de petites perceptions.

Henri F. Ellenberger précise cette distinction dans la Conférence prononcée à l'inauguration de l'hôpital Pierre Janet à Hull, le 12 septembre 1969 [96].

Maine de Biran voyait, au-dessous de la vie proprement humaine faite d'efforts conscients, une vie animale constituée par les émotions élémentaires, les instincts, les habitudes, et manifestée aussi dans le rêve et le somnambulisme. C'est précisément cette vie sous-jacente que Janet appela le "subconscient». Une remarque s'impose ici. Janet parla $d u$ subconscient, pour bien distinguer ce groupe de manifestations psychologiques de l'inconscient que la métaphysique de Schopenhauer et de von Hartmann avait mis si fort à la mode à cette époque. La définition que Janet donnait du subconscient était purement empirique : "Ces faits ont pour l'observateur qui les voit du dehors l'aspect de manifestations parfaitement intelligibles, et pour le malade chez qui elles se produisent, l'aspect de manifestations étrangères auxquelles sa personnalité ne prendrait aucune part. »Contrairement à une légende propagée par quelques psychanalystes, Janet n'a jamais renié sa conception initiale du subconscient. Encore, en 1939, Janet racontait qu'en se livrant à son passe-temps favori, la botanique, il avait trouvé une petite plante qui lui semblait tout à fait inconnue; au moment où il l'examinait à la loupe pour essayer de l'identifier par ses caractères morphologiques, sa pensée murmurait le mot " chrysospleniurn », mot qui se trouva être le nom de cette plante. Or, il lui semblait certain de n'avoir jamais vu cette plante ni entendu ce nom. La seule explication plausible était qu'il avait dî voir un jour, dans quelque ouvrage de botanique, un dessin de cette plante avec son nom latin. Il s'agissait évidemment d'une résurgence d'un souvenir subconscient. Mais Janet persistait à affirmer que les activités subconscientes de l'esprit doivent être explorées avec prudence et qu'il ne faut jamais rien affirmer sans en être sûr.

L'inconscient, lorsqu'il se dissocie du subconscient, forme alors ces idées fixes, obsessions, phobies qui peuvent se durcir sur une longue durée en névrose, c'est-à-dire, pour Janet, en un arrêt dans l'évolution des tendances : à savoir, par exemple, le refus de modifier son comportement, ce qui impliquera de se réfugier dans des postures anciennes, à la limite du dédoublement de personnalité qui se caractérise par la psychose, qu'elle soit paranoïaque ou schizophrénique, c'est-à-dire, selon Janet, la dissociation elle-même. En effet, une partie de la personnalité s'effraie de ce que fait l'autre partie, jusqu'à ce que des mouvements s'effectuent sans que la synthèse ou conscience de la personnalité puisse s'en rendre compte.

Une telle problématisation des enjeux psychiques montre bien que nous sommes bien loin de la petite comédie mondaine brossée hâtivement par une Elisabeth Roudinesco, soucieuse de rabaisser Janet, uniquement brossé dans son écrit comme l'envieux d'un Freud au firmament des questions fondamentales...

\section{Notes}

1. Nuttin J (1980) Théorie de la motivation humaine. Paris : PUF, p. 26

2. Jacob O (2000) Paris

3. Idem, p. 53

4. Idem, p. 272

5. (1975) De l'angoisse à l'extase. Paris (1926) : éditions Société Pierre-Janet., T.II, p. 119;134-135

6. (2004) Ibidem., T.I, p. 325, et T.II, p. 228. Voir également Leçons au Collège de France, 1895-1934, (1917,17, 64-70). Paris : L'Harmattan, p. 94

7. 1952. Rappelons que dans un passage de la revue Critique, 58, mars 1952, pp. 272-278, intitulé "Notes. Vue d'ensemble sur le mysticisme », Georges Bataille avait noté que parmi les patients de Pierre Janet considérés comme mystiques, il y avait un certain Martial qui n'était autre que Raymond Roussel, selon l'article de Frédéric Keck, Le primitif et le mystique chez Lévy-Bruhl, Bergson et Bataille, note 31: http://methodos.revues.org/document111.html

8. Pierre Janet le cite dans De l'angoisse à l'extase, op.cit., T.I, pp. 115-118, et T.II, p. 164

9. Ibidem., T.II, p. 255

10. Ibidem., p. 272. Voir également La tension psychologique et ses oscillations, in Traité de Psychologie. Paris : éditions Librairie Félix Alcan, 1923, Tome I, chapitre IV, I, L'automatisme des tendances, pp. 939-940. Voir également De l'angoisse à l'extase, op.cit., T.II, pp. 92 et suivantes

11. (1926) De l'angoisse à l'extase. Paris : éditions Société PierreJanet, 1975, T.I, pp. 176-179

12. Janet $P$ (1903) Les obsessions et la psychasthénie. Paris : $2^{\mathrm{e}}$ partie, $3^{\mathrm{e}}$ section, 2, l'hypothèse de la dérivation psychologique, nouvelle édition l'Harmattan, 2005, Tome II, p. 559

13. (1913) La psychoanalyse. In La psychanalyse de Freud. Paris, 2004, II, Le mécanisme pathologique du souvenir traumatique, pp. $75-76$

14. Janet P (1990) Névroses et idées fixes (1898) Paris: éditions Société Pierre-Janet, p. 393 
15. De l'angoisse à l'extase, op.cit, T.II, p. 75

16. Ibidem., p. 131;389

17. Leçons au Collège de France, op.cit., Le degré d'activation des tendances, p. 95

18. De l'angoisse à l'extase, op.cit, T.II, p. 135-136

19. Ibidem., p. 326

20. Ibidem., pp. 77, 202, 421

21. Psychologie des sentiments de M. Pierre Janet, Revue de métaphysique et de morale, Paris, 1933, XL, p. 526

22. De l'angoisse à l'extase, op.cit, leur explication remplit la totalité du T.II

23. Janet $\mathrm{P}$ (2003) Conférences à la Salpêtrière, (1892) Paris : L'Harmattan, pp. 33-36. Également L'automatisme psychologique, (1889), Paris, Odile Jacob, 1994, p. 229. Et De l'angoisse à l'extase, op.cit., T.II, pp. 195-199

24. De l'angoisse à l'extase, op.cit, T.II, p. 163

25. De l'angoisse à l'extase, op.cit., T.II, p. 92

26. De l'angoisse à l'extase, op.cit., T.I, p. 315

27. De l'angoisse à l'extase, op.cit., T.II, p. 272. Voir également $\mathrm{La}$ tension psychologique et ses oscillations, in Traité de Psychologie, op.cit., p. 939

28. Janet, Ibidem, pp. 945-946. Voir également De l'angoisse à l'extase, op.cit., T.II, p. 321, en particulier, pp. 323 et suivantes

29. Janet, La tension psychologique et ses oscillations, in Traité de Psychologie, op.cit., p. 923. Voir également De l'angoisse à l'extase, op.cit., T.II, p. 420

30. De l'angoisse à l'extase, op.cit., T.I, p. 254

31. Janet, Névroses et idées fixes, (1898), réédition Paris, 1990, Société Pierre-Janet, p. 393

32. De l'angoisse à l'extase, op.cit., T.I, p. 258

33. Ibidem, p. 252

34. Ibidem, p. 266

35. Ibidem, pp. 268-269

36. Ehrenberg, op.cit., 2000, p. 52-60;273

37. Ibidem, p. $59 ; 274$

38. Discrepancy ou discrépance, je suis ici l'utilisation que fait Joseph Nuttin de ce terme lorsqu'il explique dans son livre phare, Théorie de la motivation humaine, (op.cit., 1980, p. 239), que de "préférence aux termes voisins de divergence, discordance, dissonance ou incongruence, nous utiliserons souvent, dans la suite de cet ouvrage, le vocable "discrépance", transposition littérale du mot discrepancy, largement répandu dans la littérature psychologique anglo-saxonne. Discrépance nous semble, en effet, indiquer de façon plus technique cette notion dynamique d'écart entre deux données à laquelle nous faisons ici référence ", parce qu'il s'agit, pour Nuttin, de souligner que cet écart semble permanent en tant que constante constitutive de l'action et non pas comme effet pervers extérieur à son émergence.

39. Ehrenberg, op.cit., p. 274

40. De l'angoisse à l'extase, op.cit., T.I, p. 266

41. La Fatigue d'être soi, op.cit., p. 274

42. De l'angoisse à l'extase, op.cit., T.II, p. 325

43. La Fatigue d'être soi, op.cit., p. 274

44. Ibidem, pp. 254-258

45. La Fatigue d'être soi, op.cit., p. 272

46. Les névroses, Paris, 1909, seconde partie, 4. Les névroses, maladies et l'évolution des fonctions, p. 323

47. Ibidem, p. 323

48. La Fatigue d'être soi, op.cit., p. 53

49. Ibidem, p. 223

50. Ibidem, p. 227
51. Ibidem, p. 210

52. Ibidem, p. 195

53. Ibidem, p. 231

54. Ibidem, p. 232

55. Ibid., T.II, pp. 85-88, et pp. 195-196

56. Ibidem., p. 134

57. Ibidem., p. 199

58. Ibidem p. 198

59. Ibid, T. I, p. 223

60. Ibidem, p. 209

61. Ibidem, p. 214

62. Ibidem, p. 244

63. Ibidem, pp. 208; 270

64. Par exemple dans Le juste et le vrai, Paris, Fayard, 1985, p. 167. Et dans Renouveler la démocratie, Odile Jacob, Paris, 2006, pp. 202-204

65. De L'angoisse à l'extase, op.cit., T.I, p. 207

66. Ibidem., pp. 176; 315

67. Ibidem., p. 195

68. (1925) éditions Gallimard, Paris, collection Les Essais, 1949, p. 31

69. (2004) Janet, La psychanalyse de Freud, (1913). Paris : L'Harmattan, p. 58

70. (2003) Serge Nicolas, introduction aux Conférences à la Salpêtrière. Paris : L'Harmattan, p. 7

71. Serge Nicolas, op.cit., Conférences à la Salpêtrière..., p. 14

72. Ma vie...op.cit., p. 25

73. op.cit., pp. 386-389

74. (2004) Janet, La psychanalyse de Freud, (1913) Paris : L'Harmattan, pp. 100-103

75. Jones E (1911) « The Pathology of Morbid Anxiety », Journal of Abnormal Psychology, juillet, p. 98

76. Ibidem., pp. 17-18

77. Ibidem, p. 28

78. Serge Nicolas, introduction aux Conférences à la Salpêtrière, op.cit., p. 9

79. Freud, Ma vie... op.cit., p. 17

80. Ibidem

81. Idem, p. 19

82. Ibidem

83. Nous reviendrons sur ce point dans un prochain travail

84. Janet, La psychanalyse de Freud, op.cit., p. 58

85. Ibidem, p. 75

86. Janet, De l'angoisse à l'extase, op.cit., T.II, p. 255

87. (1994) Paris : Fayard, op.cit., Tome 1, p. 243

88. Ibidem, pp. 224-225

89. (1972) Introduction à la psychanalyse, (1916). Paris : éditions petite bibliothèque Payot, p. 317

90. Bouchara C (2006) Hypnose sous le pas de Charcot, in Synapse, $\mathrm{n}^{\mathrm{o}} 221$, p. 38

91. Idem, p. 37

92. (2004) La psychoanalyse (1913), In: La psychanalyse de Freud, Paris, II, Le mécanisme pathologique du souvenir traumatique, pp. $75-76$

93. Janet, La tension psychologique et ses oscillations, in Traité de Psychologie, op.cit., p. 923. Voir également De l'angoisse à l'extase, op.cit., T.II, p. 420

94. Janet, Névroses et idées fixes, op.cit., p. 393

95. Naccache L. Le nouvel inconscient, Freud, Christophe Colomb des neurosciences. Paris : éditions Odile Jacob, pp. 335-337

96. (1994) Ellenberger Histoire de la découverte de l'inconscient, Fayard, p. 400 